\title{
Alterstice
}

Revue internationale de la recherche interculturelle

International Journal of Intercultural Research

Revista International de la Investigacion Intercultural

\section{Vers une conception théorique multidimensionnelle du climat scolaire interculturel}

\author{
Isabelle Archambault, Marie Mc Andrew, Geneviève Audet, Corina \\ Borri-Anadon, Sivane Hirsch, Valérie Amiraux et Kristel Tardif-Grenier
}

Volume 8, numéro 2, 2018

L'école à l'épreuve de la diversité ethnoculturelle de son personnel : regards compréhensifs croisés

URI : https://id.erudit.org/iderudit/1066957ar

DOI : https://doi.org/10.7202/1066957ar

Aller au sommaire du numéro

Éditeur(s)

Alterstice

ISSN

1923-919X (numérique)

Découvrir la revue

Citer cet article

Archambault, I., Mc Andrew, M., Audet, G., Borri-Anadon, C., Hirsch, S., Amiraux, V. \& Tardif-Grenier, K. (2018). Vers une conception théorique multidimensionnelle du climat scolaire interculturel. Alterstice, 8(2), 117-132. https://doi.org/10.7202/1066957ar
Résumé de l'article

Le climat d'une école résulte des relations continues entre les individus qui la composent. Dans les milieux où la diversité ethnoculturelle est très présente, ces relations entre les acteurs contribuent donc nécessairement à la construction du climat. Il n'existe toutefois aucune définition claire du climat scolaire interculturel. Une telle définition est pourtant nécessaire, tant pour mieux comprendre les normes, les valeurs, les croyances et les échanges qui existent entre les acteurs issus de la diversité au sein d'un milieu que pour identifier les pratiques qui sont ou qui devraient être mises en place par l'école pour soutenir la réussite de tous les élèves. S'inscrivant dans une perspective interculturelle et inclusive, nous visons dans un premier temps à combler les limites actuelles des écrits scientifiques en proposant une définition théorique du climat scolaire interculturel qui prévaut dans les écoles. Cette définition comporte cinq dimensions, soit 1) l'engagement de l'école en faveur d'une culture d'équité et d'ouverture à la diversité dans les rapports avec les élèves, les familles et la collectivité, 2) le statut et la légitimité des cultures et des langues d'origine dans les pratiques en classe et dans les normes et les règlements des établissements, 3 ) les attitudes du personnel à l'égard des élèves et des familles d'origines diverses et de la diversité en général, 4) la qualité des relations interculturelles entre les élèves et le personnel d'origines diverses et, enfin, 5) le soutien de l'école à la construction identitaire des jeunes issus de la diversité. Dans un second temps, nous présentons un portrait des rares liens documentés entre ces différentes facettes du climat scolaire interculturel et la réussite éducative des élèves.
(C) Isabelle Archambault, Marie Mc Andrew, Geneviève Audet, Corina Borri-Anadon, Sivane Hirsch, Valérie Amiraux et Kristel Tardif-Grenier, 2018
Ce document est protégé par la loi sur le droit d'auteur. L’utilisation des services d'Érudit (y compris la reproduction) est assujettie à sa politique d'utilisation que vous pouvez consulter en ligne.

https://apropos.erudit.org/fr/usagers/politique-dutilisation/ 


\section{7}

alterstice

Revue Internationale

de la Recherche Interculturelle

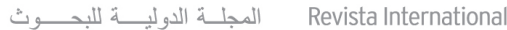

de la Investigacion Intercultural
Revista Internacional de Pesquisa Intercultural
International Journal of Intercultural Research

ARTICLE THÉMATIQUE

\section{Vers une conception théorique multidimensionnelle du climat scolaire interculturel}

Isabelle Archambault ${ }^{1}$, Marie Mc Andrew ${ }^{1}$, Geneviève Audet ${ }^{2}$, Corina Borri-Anadon ${ }^{3}$, Sivane Hirsch ${ }^{3}$, Valérie Amiraux ${ }^{1}$ et Kristel Tardif-Grenier ${ }^{4}$

\section{Résumé}

Le climat d'une école résulte des relations continues entre les individus qui la composent. Dans les milieux où la diversité ethnoculturelle est très présente, ces relations entre les acteurs contribuent donc nécessairement à la construction du climat. II n'existe toutefois aucune définition claire du climat scolaire interculturel. Une telle définition est pourtant nécessaire, tant pour mieux comprendre les normes, les valeurs, les croyances et les échanges qui existent entre les acteurs issus de la diversité au sein d'un milieu que pour identifier les pratiques qui sont ou qui devraient être mises en place par l'école pour soutenir la réussite de tous les élèves. S'inscrivant dans une perspective interculturelle et inclusive, nous visons dans un premier temps à combler les limites actuelles des écrits scientifiques en proposant une définition théorique du climat scolaire interculturel qui prévaut dans les écoles. Cette définition comporte cinq dimensions, soit 1) l'engagement de l'école en faveur d'une culture d'équité et d'ouverture à la diversité dans les rapports avec les élèves, les familles et la collectivité, 2) le statut et la légitimité des cultures et des langues d'origine dans les pratiques en classe et dans les normes et les règlements des établissements, 3) les attitudes du personnel à l'égard des élèves et des familles d'origines diverses et de la diversité en général, 4) la qualité des relations interculturelles entre les élèves et le personnel d'origines diverses et, enfin, 5) le soutien de l'école à la construction identitaire des jeunes issus de la diversité. Dans un second temps, nous présentons un portrait des rares liens documentés entre ces différentes facettes du climat scolaire interculturel et la réussite éducative des élèves.

\section{Rattachement des auteurs}

${ }^{1}$ Université de Montréal, Montréal, Canada ; ${ }^{2}$ Université du Québec à Montréal, Montréal, Canada ; ${ }^{3}$ Université du Québec à Trois-Rivières, Trois-Rivières, Canada ; ${ }^{4}$ Université du Québec en Outaouais, Gatineau, Canada

\section{Correspondance}

isabelle.archambault@umontreal.ca

\section{Mots clés}

climat scolaire interculturel, inclusion, équité, diversité ethnoculturelle, réussite éducative

\section{Pour citer cet article}

Archambault, I., Mc Andrew, M., Audet, G., Borri-Anadon, C., Hirsch, S., Amiraux, V. et Tardif-Grenier, K. (2018). Vers une conception théorique multidimensionnelle du climat scolaire interculturel. Alterstice, 8(2), $117-132$. 


\section{Introduction}

Le climat scolaire est une variable importante dans l'expérience scolaire des élèves, qui contribue à leur réussite éducative (Janosz, Georges et Parent, 1998). Produit des interactions sociales entre tous les acteurs qui fréquentent et animent l'école (Janosz, Georges et Parent, 1998), il est souvent associé à la " personnalité » d'un milieu. La diversité ethnoculturelle, lorsqu'elle est présente, se reflète donc logiquement dans le climat scolaire. Pourtant, la dimension interculturelle est rarement prise en compte pour décrire ou analyser les différentes facettes du climat scolaire, voire identifier son impact sur la réussite des élèves. Cet article constitue une première tentative de combler cette lacune.

Dans cette perspective, nous avons assigné deux objectifs principaux à cet article. Dans un premier temps, nous proposons une formulation théorique des différentes dimensions du climat scolaire interculturel qui prévaut dans les écoles, allant de dimensions plus systémiques aux dimensions plus individuelles. Dans un second temps, nous présentons une cartographie des liens documentés entre ces différentes facettes du climat interculturel et la réussite éducative des élèves issus de la diversité. Cette réflexion théorique repose à la fois sur une revue exhaustive des études scientifiques réalisées au Québec au cours des dernières années sur l'expérience scolaire des élèves issus de l'immigration (Mc Andrew et collab., 2015) et sur une recension des études québécoises et internationales portant sur le climat scolaire ou la réussite éducative des élèves.

\section{Qu'entend-on par le climat scolaire?}

Défini de multiples façons dans la littérature scientifique, le climat scolaire renvoie aux normes, valeurs et croyances qui caractérisent une école, ainsi qu'aux caractéristiques de l'environnement éducatif, social et interpersonnel du milieu (Cohen, McCabe, Michelli et Pickeral, 2009 ; Janosz, Georges et Parent, 1998 ; Kuperminc, Leadbeater, Emmons et Blatt, 1997 ; Kuperminc, Leadbeater, Loukas et Robinson, 2004 ; White, La Salle, Ashby et Meyers, 2014). Il s'agit donc de la résultante d'interactions entre les individus et leur environnement, découlant des caractéristiques, des perceptions et des relations qui prévalent entre les nombreux acteurs impliqués dans la vie d'un établissement. On citera, par exemple, l'interaction entre les élèves, les membres du personnel, les parents et des représentants de la collectivité avec les composantes physiques et organisationnelles du milieu, comme la qualité des services et des infrastructures (Janosz, Georges et Parent, 1998).

De façon générale, la recherche suggère que le climat d'une école marque de manière significative l'expérience scolaire et le bien-être des acteurs qui la fréquentent (La Salle, 2018). La présence d'un " climat positif » au sein d'une école est ainsi associée à de nombreuses conséquences favorables chez les élèves, tant sur le plan de leur réussite scolaire, de leur engagement en classe et à l'école, de leur sentiment de sécurité, que de leur santé psychologique et de leur bien-être en général (Bradshaw, Koth, Thornton et Leaf, 2009 ; Konishi, Miyazaki, Hymel et Waterhouse, 2017 ; La Salle, George, McCoach, Polk et Evanovich, 2018 ; Leadbeater, Sukhawathanakul, Thompson et Holfeld, 2015 ; Wang, Boyanton, Ross, Liu, Sullivan et Do, 2018).

\section{Pourquoi s'intéresser au climat interculturel ?}

II n'existe à ce jour aucune définition claire du climat scolaire tenant compte de la diversité ethnoculturelle présente dans plusieurs milieux. Pourtant, le climat d'une école résulte des relations continues entre les individus qui la composent (Janosz, Georges et Parent, 1998). Les rapports entre les acteurs issus de la diversité ethnoculturelle au sein d'une même école ne peuvent donc que participer à la construction du climat et, de ce fait, doivent être pris en compte dans sa définition. Mais qu'est-ce que la diversité ethnoculturelle ? Cet état de fait mesurable renvoie à différents marqueurs (tels l'origine, la langue et la religion) qui participent tant aux dynamiques d'identification et de stratification sociales qu'aux représentations que se font les membres d'une société par rapport à des individus ou des groupes (Amiraux, 2018). Ainsi conçue, la diversité ethnoculturelle est historiquement une composante inhérente de presque toutes les sociétés modernes constituées par des groupes divers, majoritaires ou minoritaires, qui entretiennent des rapports de pouvoir complexes et la plupart du temps inégalitaires. Au Canada, les représentants des Premières Nations, les francophones et certaines minorités religieuses ou "racisées" de longue date sont souvent confrontés à ces rapports inégalitaires. La diversité 
ethnoculturelle est néanmoins souvent ramenée à la présence de populations issues de l'immigration, c'est-à-dire aux individus nés à l'étranger ou nés au Canada de parents nés à l'étranger.

L'accroissement de la diversité ethnoculturelle dans les écoles en Occident, y compris hors des grands centres urbains (Borri-Anadon et Hirsch, 2019; Gélinas, Hirsch et Borri-Anadon, soumis), semble ainsi appeler au développement d'une définition « interculturelle » du climat scolaire. Une telle définition est d'ailleurs cohérente avec la direction prise, depuis le milieu des années 1970, par les énoncés de politiques interculturelles qui soulignent la nécessité de prendre en compte la diversité en éducation (Mc Andrew, 2001 ; Milot, 2014). Dans une perspective interculturelle et inclusive, comprendre les dynamiques scolaires passe en effet par la prise en compte des caractéristiques ethnoculturelles des groupes en présence et par une analyse exhaustive de l'impact de cette diversité sur la construction du climat scolaire. Une vision interculturelle du climat scolaire peut notamment mettre en lumière les relations qui existent entre les individus de différents groupes ethnoculturels au sein de l'école et permettre de mieux saisir tant leurs perceptions, attitudes et comportements que les pratiques qui sont ou qui devraient être mises en place par l'école pour les soutenir. Le climat scolaire interculturel se définit ainsi comme la résultante des relations et des échanges qui s'instaurent entre les individus issus de la diversité ethnoculturelle au sein d'une même école, jeunes et adultes, incluant les rapports entre les individus formant les groupes minoritaires et ceux formant le groupe majoritaire, en interaction avec les caractéristiques, les composantes physiques et organisationnelles et les pratiques mises en place dans le milieu.

Les études qui ont évalué le climat scolaire interculturel sont quasi inexistantes. Néanmoins, quelques études transnationales suggèrent que, bien que le climat scolaire soit souvent défini de la même façon dans différents pays (Jia et collab., 2009 ; La Salle, Meyers, Varjas et Roach, 2015), il peut être perçu différemment dans l'école, en fonction des caractéristiques ethnoculturelles des groupes en présence (Voight, Hanson, O’Malley et Adekanye, 2015). Aux États-Unis et en Europe, certains travaux indiquent par exemple que les élèves issus de la diversité tendent à évaluer le climat de leur école comme étant moins sécuritaire et soutenant comparativement à leurs pairs du groupe majoritaire (McNeely, Nonnemaker et Blum, 2002 ; Watkins et Aber, 2009).

\section{Le climat interculturel : une notion multidimensionnelle}

Le climat scolaire est généralement défini par plusieurs dimensions, qui renvoient principalement aux trois grandes sphères proposées par Moos (1979): le système d'encadrement et les pratiques du milieu, les relations qui y prévalent et la croissance personnelle des individus qui le fréquentent. Dans la plupart des études du climat scolaire, ce cadre théorique multidimensionnel prévaut (Janosz, Georges et Parent, 1998 ; Parris, Rocha Neves et La Salle, 2018 ; Sanders, Durbin, Anderson, Fogarty, Giraldo-Garcia et Voight, 2018). Le climat scolaire interculturel a donc avantage à être également appréhendé dans une perspective multidimensionnelle, afin de prendre en compte la complexité des objets que le concept recouvre. Cependant, à l'exception de quelques travaux sur l'acceptation culturelle ou le respect de la diversité comme sous-facettes du climat relationnel de l'école (La Salle, 2018 ; Wang et collab., 2018), les différentes dimensions du climat scolaire en place ne sont pas abordées sous l'angle interculturel, limitant ainsi la compréhension que les chercheurs et les acteurs éducatifs peuvent avoir de l’impact de la diversité à l'école.

Mc Andrew et ses collègues ont réalisé en 2015 une méta-analyse des travaux québécois sur la réussite éducative des élèves issus de l'immigration. Ce travail a permis d'identifier plusieurs des enjeux spécifiques à l'expérience socioscolaire de ces élèves et de faire ressortir certaines spécificités des établissements qu'ils fréquentent. Nous basant sur cette méta-analyse, nous proposons ici une définition théorique multidimensionnelle du climat interculturel qui articule, d'une part, les enjeux soulevés par Mc Andrew et, d'autre part, les trois grandes sphères du climat éducatif de Moos (1979). Nous dégageons ainsi cinq dimensions du climat scolaire interculturel, allant des plus systémiques aux plus individuelles, soit 1) l'engagement de l'école en faveur d'une culture d'équité et d'ouverture à la diversité dans les rapports avec les élèves, les familles et la collectivité, 2) le statut et la légitimité des cultures et des langues d'origine dans les pratiques en classe et dans les normes et les règlements des établissements, 3) les attitudes du personnel à l'égard des élèves et des familles d'origines diverses et de la diversité en général, 4) la qualité des relations interculturelles entre les élèves et le personnel d'origines diverses et, enfin, 5) le soutien de l'école à la construction identitaire des jeunes issus de la diversité. 
Ces cinq dimensions du climat scolaire s'inscrivent dans une perspective interculturelle et inclusive qui souligne l'importance de reconnaître les réalités spécifiques des élèves issus de la diversité, d'adopter des pratiques d'équité à leur endroit et de favoriser le vivre ensemble (Larochelle-Audet, Magnan, Potvin et Doré, 2018 ; Potvin, Larochelle-Audet, Campbell, Kingué-Élonguélé et Chastenay, 2015). Chacune de ces dimensions du climat est donc essentielle et toutes peuvent influencer tant la représentation que les acteurs scolaires ont de leur école que la propension de l'établissement à être "inclusif ", c'est-à-dire à s'adapter aux besoins de tous ses élèves, à mettre à profit la diversité sous toutes ses formes et à favoriser un accès équitable et les mêmes chances pour tous de s'épanouir (Conseil supérieur de l'éducation, 2017). Comme nous en ferons la démonstration en deuxième partie, ces cinq dimensions sont également liées à la réussite éducative des élèves de différents groupes ethnoculturels, dont ceux issus de l'immigration. Leur examen attentif, tant par les chercheurs que les praticiens, ne peut que contribuer à orienter la façon dont les décideurs et les intervenants scolaires peuvent s'engager dans le développement ou la mise en œuvre d'encadrements, de politiques, de programmes ou de pratiques favorables à la réussite scolaire de tous les élèves (Mc Andrew et collab., 2015). Les sections qui suivent définissent les cinq dimensions du climat interculturel autour desquelles s'articule notre proposition théorique.

\section{Dimension 1. L'engagement en faveur d'une culture d'équité et d'ouverture à la diversité dans les rapports avec les élèves, les familles et la collectivité}

L'engagement d'une l'école en faveur d'une culture d'équité et d'ouverture à la diversité peut se traduire de multiples façons. D’abord, dans son projet éducatif, son code de vie, ses pratiques quotidiennes dans l'école et dans les classes. Ensuite, dans les partenariats que l'établissement entretient avec les familles et les organismes communautaires ou religieux qui interviennent directement avec les jeunes issus de l'immigration et leurs familles. Certaines écoles semblent toutefois réussir mieux que d'autres à soutenir cet engagement. Ces établissements mettent notamment de l'avant des politiques de tolérance zéro à l'égard de la discrimination ou du racisme, en assurant une visibilité des indicateurs relatifs à la réussite chez les minorités (ethnic monitoring), en incitant la participation des familles immigrantes ou en établissant des partenariats égalitaires avec divers organismes communautaires représentant des groupes minoritaires (Mc Andrew et collab., 2015). De manière plus concrète, certaines initiatives, comme les jumelages entre parents francophones et allophones, la traduction en diverses langues du code de vie, le développement de capsules pédagogiques sur l'immigration ou encore la prise en compte des fêtes religieuses, ont été documentées pour leurs retombées positives (Comité consultatif sur l'intégration et l'accommodement raisonnable en milieu scolaire [CCIARMS], 2007). Des recherches québécoises suggèrent d'ailleurs que nombre de parents et d'élèves issus de l'immigration considèrent l'école comme un milieu généralement ouvert aux familles, même si certaines tensions liées à des incompréhensions culturelles peuvent parfois être identifiées. Les partenariats avec le secteur communautaire sont fréquents, même si on note plus de réticence de la part des écoles à s'engager et à établir des partenariats avec des organismes liés à des communautés spécifiques ou à vocation religieuse (Audet, Mc Andrew et Lamothe-Lachaîne, soumis; Harnois, 2010 ; Lafortune, 2012 ; Mc Andrew, Ephraïm, Lemire et Swift, 2004 ; Rahm, 2013 ; Vatz Laaroussi, Rachédi, Kanouté et Duchesne, 2005).

En dépit de succès réels, certains élèves et parents appartenant à des communautés marginalisées ou stigmatisées (tels les Antillais ou les musulmans par exemple) rapportent plus souvent des comportements ou des pratiques à effet d'exclusion de la part des intervenants scolaires (Bakhshaei, 2013 ; Livingstone, Celemencki et Calixte, 2014 ; Triki-Yamani et Mc Andrew, 2009). Un autre enjeu lié à l'équité, et dont le lien est encore plus étroit avec la réussite scolaire, est celui de l'évaluation et du classement des élèves issus de l'immigration. L'importance de la reconnaissance des acquis à l'arrivée et d'une évaluation équitable tout au long de la scolarité fait l'objet d'un large consensus (Alspaugh, 1998 ; Lucas, 2001), pourtant, dans la réalité, l'application de mesures ou de façons de faire adaptées demeure relativement rare. Plusieurs études confirment l'existence de problèmes significatifs dans l'évaluation et dans le classement, et qui affectent tout particulièrement certains élèves. On pense notamment aux nouveaux arrivants qui fréquentent les classes d'accueil, aux élèves issus de l'immigration dont les difficultés d'apprentissage sont pressenties, ainsi que les élèves arrivés en cours de scolarité qui terminent leur secondaire à l'éducation des adultes (Borri-Anadon, 2014 ; De Koninck et Armand, 2012 ; Potvin et collab., 2015). 


\section{Dimension 2. Le statut et la légitimité des cultures et des langues d'origine dans les normes et pratiques}

Le statut et la légitimité des cultures et des langues d'origine dans les normes et les pratiques scolaires est une deuxième dimension du climat interculturel qui peut se traduire non seulement par des orientations concrètes dans l'école en matière linguistique, mais également par l'acceptation de ces choix par les différents acteurs du milieu scolaire (Armand, 2013). Les écoles qui valorisent les cultures et langues d'origine autorisent leurs élèves à utiliser une langue autre que celle de scolarisation dans des contextes variés, ailleurs que dans les cours de langue ou en classe d'accueil. Ces écoles mettent également en place des activités qui valorisent et favorisent le multilinguisme, tout en accordant de la place à la diversité culturelle, linguistique, religieuse et à l'éducation interculturelle dans différentes activités autres que les cours où ce contenu fait partie du programme. Cette ouverture se manifeste de plusieurs façons, comme l'affichage dans les couloirs de travaux ou de messages valorisant la diversité, ainsi que le fait de souligner les fêtes de traditions religieuses et culturelles diverses. En classe, on citera l'emploi de matériel didactique reflétant les cultures des élèves ainsi que la mise en place d'activités pédagogiques valorisant le multilinguisme, la diversité ou la contribution de communautés spécifiques à I'histoire dite nationale. Ces initiatives favorisant le développement d'attitudes positives face à la diversité semblent contribuer au développement identitaire des élèves (Armand et Maraillet, 2013; Armand, Hardy et Lemay, 2014).

En pratique, la situation québécoise au regard de cette dimension est mitigée. D'un côté, plusieurs études soulignent la progression notable de la représentation des cultures minoritaires dans le matériel didactique et dans les programmes (Oueslati, Mc Andrew et Helly, 2011 ; Hirsch et Mc Andrew, 2013 ; Triki-Yamani et Mc Andrew, 2009). De plus, dans les écoles où le pluralisme culturel et linguistique est valorisé et où sont mis en place des accommodements aux normes et règlements dans le respect des valeurs culturelles et religieuses des élèves et des membres du personnel, les effets sont généralement positifs (Mc Andrew et Audet, sous presse). Mais d'un autre côté, ces initiatives ne sont pas généralisées et la résistance vis-à-vis de la valorisation d'autres langues que le français reste forte, reflétant la vigueur des enjeux linguistiques au Québec. La diversité religieuse fait quant à elle de plus en plus l'objet de débats dans la foulée de controverses sociales plus larges qui existent internationalement (Armand, 2013; Thamin, 2011; CCIARMS, 2007; Piet et Granjean, 2012). II reste donc beaucoup de travail à accomplir à l'égard de cette dimension pour que les éléments qui la composent soient mis en œuvre sur le terrain.

\section{Dimension 3. Les attitudes du personnel à l'égard de la diversité, des élèves et des familles}

Les attitudes du personnel à l'égard de la diversité renvoient tant aux perceptions et attitudes des enseignants, des professionnels ou des autres acteurs scolaires envers les élèves issus de la diversité et leurs familles qu'aux attentes qu'ils ont à l'égard de ces élèves. Cette dimension, comme la suivante, concerne l'aspect relationnel du climat scolaire. Au Québec, les études plus générales sur l'intégration scolaire des élèves issus de l'immigration en présentent un portrait mitigé (Bakhshaei, 2013 ; Borri-Anadon, 2014 ; De Koninck et Armand, 2012 ; Mc Andrew et collab., 2004 ; Murphy, 2014 ; Vatz Laaroussi et collab., 2005). En effet, si l'évaluation de la situation scolaire de ces élèves et de la valorisation de l'éducation par leurs familles est globalement positive, les attitudes et représentations des intervenants scolaires à l'égard de la diversité demeurent parfois teintées négativement. Ces représentations semblent notamment fortement influencées par les difficultés que connaissent certains nouveaux arrivants ou groupes appartenant à certaines minorités racisées. Ainsi, dans certaines écoles où la diversité ethnoculturelle est plus importante, les acteurs scolaires auraient des perceptions plus négatives et des attentes moins élevées à l'égard de leurs élèves que dans les milieux plus homogènes (Agirdag, Van Houtte et Van Avermaet, 2013 ; Brault, Janosz et Archambault, 2014).

Certains auteurs suggèrent que l'adoption de systèmes d'interprétation de l'échec scolaire axés sur la responsabilité de l'élève et de la famille ou, au contraire, sensibles aux dimensions systémiques et institutionnelles, aurait un impact sur ces attentes. En effet, cela influencerait notamment l'engagement des enseignants à soutenir la réussite des élèves à risque, l'efficacité de leurs pratiques pédagogiques et la collaboration école-famille (Benoît, Rousseau, Ngirumpatse et Lacroix, 2008; Sharkey et Layzer, 2000 ; Tardif-Grenier, Archambault, Lafantaisie et Magnan, 2018). Dans le premier cas, le fait d'attribuer les difficultés vécues par les élèves issus de l'immigration à leurs caractéristiques ou à celles des familles (par exemple, à des enjeux linguistiques, aux retards cumulés ou au 
faible capital culturel des parents) est généralement associé à une forme de désengagement du personnel scolaire, qui peut alors se sentir impuissant face aux difficultés de leurs élèves. À l'inverse, la capacité des milieux à identifier les enjeux qui relèvent de l'organisation des services ou des pratiques de l'école pouvant contribuer à créer ou à accentuer les difficultés éprouvées par certains élèves serait associée à une plus grande mobilisation de l'école vers I'identification de solutions efficaces à long terme (Mc Andrew et collab., 2015).

\section{Dimension 4. La qualité des relations interculturelles entre les élèves et le personnel d'origines diverses}

La qualité des relations interculturelles entre les élèves et le personnel d'origines diverses est une autre des facettes du climat relationnel, qui renvoie cette fois à l'intégration sociale des élèves issus de l'immigration et à la qualité des relations qu'ils entretiennent entre eux, avec leurs pairs et avec les membres du personnel de l'école. Elle implique également la qualité des relations existant entre l'ensemble des intervenants scolaires (enseignants, personnel de soutien, professionnels ou direction), dont ceux d'implantation ancienne et d'immigration récente.

Plusieurs études ont examiné les relations entre élèves d'origines diverses. Elles portent sur plusieurs sousdimensions disparates telles que la qualité des relations d'amitié, la discrimination ou l'isolement. Certains écrits suggèrent notamment que le fait d'appartenir à un groupe majoritaire au sein d'une l'école ou de s'affilier à des pairs de la même origine ethnique que soi résulte en une discrimination moindre et un meilleur engagement scolaire, surtout lorsque ces pairs valorisent aussi l'école (Bingham et Okagaki, 2012 ; Fuligni, 1997; Ryan, 2000 ; Vaquera, 2009). II a également été démontré que le fait d'avoir de bonnes relations avec les pairs constitue une ressource très efficace pour soutenir l'intégration scolaire des élèves, particulièrement de ceux issus de l'immigration. De fait, avoir des amis qui ont une bonne connaissance de la langue, des valeurs et des codes qui prédominent dans une culture donnée peut favoriser chez l'élève issu de l'immigration une meilleure compréhension de cette culture et lui offrir une source importante de soutien pour s'intégrer et comprendre certains termes essentiels pour l'apprentissage (Suarez-Orozco, Rhodes et Milburn, 2009 ; Way, Becker et Greene, 2006).

Parallèlement, les études suggèrent que le fait d'entretenir de bonnes relations avec les enseignants semble particulièrement important pour les élèves issus de l'immigration, et notamment pour les élèves de première génération qui ont moins de repères dans le système scolaire du pays d'accueil (Lamanque-Bélanger, 2018). Si le fait d'être issus ou non de l'immigration n'a a priori aucune incidence sur les relations que les élèves entretiennent avec les adultes de l'école (Ewing et Taylor, 2009 ; Vedder, Boekaerts et Seegers, 2005), les élèves immigrants semblent réellement bénéficier du soutien des enseignants et d'autres membres du personnel scolaire, qui peuvent les guider dans leur compréhension de l'école et de ses normes (D'Amato, 1993 ; Heath, 1983). Ce soutien, qui est aussi bénéfique sur le plan des apprentissages, peut même protéger les élèves face à différentes formes de discrimination (Faircloth et Hamm, 2005 ; Garcia-Reid, 2007 ; Garcia-Reid, Reid et Peterson, 2005). Certains auteurs rapportent cependant que les acteurs scolaires seraient susceptibles d'adopter des attitudes plus positives, d'être plus compréhensifs et tolérants avec les élèves ayant le même profil ethnoculturel qu'eux (Dee, 2004 ; Glock, Kneer et Kovacs, 2013). La proportion d'enseignants immigrants ou appartenant à des minorités ethnoculturelles ou des groupes racisés étant souvent relativement faible dans plusieurs écoles, il pourrait s'agir là d'une piste expliquant certaines des difficultés relatées par les élèves de ces groupes dans leurs relations avec les enseignants (Chiu, Pong, Mori et Chow, 2012 ; Roscigno et Ainsworth-Darnell, 1999).

Enfin, même si cet enjeu émerge de plus en plus comme problématique dans les propos des gestionnaires scolaires (Institut du Nouveau Monde, 2013), les études sur le climat des relations entre les membres du personnel d'implantation plus ancienne et leurs collègues formés à l'étranger, d'arrivée plus récente ou de minorités racisées, sont relativement rares. Les quelques travaux qui existent relèvent que les acteurs scolaires ont surtout tendance à adopter une perspective déficitaire face à leurs collègues formés à l'étranger, en mettant davantage l'accent sur les difficultés rencontrées par ces derniers plutôt que sur les facteurs systémiques, qui relèvent de l'école et du système éducatif, aptes à mieux les soutenir (Morrissette, Diédhiou et Charara, 2014 ; Niyubahwe, Mukamurera et Jutras, 2013). Des recherches récentes portent toutefois un regard renouvelé et plus positif sur cet enjeu central dans le débat sur la diversité à l'école (Morrissette et Demazière, 2018). 


\section{Dimension 5. Le soutien à la construction identitaire des jeunes issus de l'immigration}

L'identité renvoie au sentiment d'appartenance que l'individu exprime par rapport à l'un ou plusieurs des groupes sociaux auxquels il s'identifie. Chez les immigrants particulièrement, cette identité est en constante évolution (Timotijevic et Breakwell, 2000). Lorsqu'elle est associée à une culture en particulier, on parle alors d'identité culturelle, c'est-à-dire de l'ensemble des éléments d'une culture par lesquels un individu ou un groupe se définissent ou se distinguent des autres (Abou, 1986). Pour les élèves issus de la diversité, il est aussi souvent question d'identité pluri- ou multi-culturelle, qui renvoie à la capacité individuelle de se définir et de s'accepter en tant que membres de plus d'un groupe ethnoculturel (Marks, Patton et Coll, 2011).

Les écoles et les acteurs scolaires qui soutiennent le développement d'une identité multiculturelle chez les élèves issus de l'immigration proposent différentes mesures, projets, initiatives ou façons de faire qui nourrissent ces jeunes et les aide à concilier leur(s) culture(s) d'origine et d'appartenance, notamment celle de leur société d'accueil. Des études ont en effet rapporté que pour ces élèves, l'engagement dans le multiculturalisme et dans le développement de compétences multiculturelles, c'est-à-dire d'habiletés leur permettant de satisfaire aux attentes et aux exigences de deux cultures et d'y vivre des succès, serait associé à des conséquences plus positives (par exemple la diminution des conflits avec leurs parents), ainsi qu'à une meilleure intégration scolaire et sociale (Miranda, Estrada et Firpo-Jimenez, 2000 ; Smokowski, Rose et Bacallao, 2008).

Plusieurs travaux suggèrent par ailleurs que soutenir adéquatement les élèves issus de la diversité passe par un accompagnement par les acteurs scolaires d'une construction identitaire multiculturelle harmonieuse. Celle-ci repose sur des stratégies par cohérence complexe qui visent la recherche d'un équilibre entre leur culture d'origine et la culture du pays d'accueil, plutôt que sur des stratégies par cohérence simple, qui les amènent à rejeter l'un ou l'autre de ces deux pôles (Camilleri, 1990 ; Hohl et Normand, 1996). Faire la promotion et favoriser l'accès à des activités parascolaires pertinentes, qui peuvent agir comme espace de réparation des tensions liées à la migration, est une première forme d'initiative pouvant favoriser l'épanouissement et l'intégration des élèves (Mc Andrew et collab., 2015). Le fait de soutenir le développement de compétences multiculturelles en permettant aux élèves de connaître et comprendre les valeurs, croyances, rites et pratiques de la culture d'accueil tout en se sentant à l'aise et libres de communiquer et de faire valoir leur culture d'origine est également à privilégier (LaFramboise, Coleman et Gerton, 1993).

En somme, le portrait des dimensions du climat scolaire interculturel ici proposé permet de mettre en évidence les relations qui existent entre les individus de différents groupes ethnoculturels au sein d'une école. Ce portrait favorise également une meilleure compréhension tant des attitudes et comportements de ces personnes que des pratiques qui doivent être mises en place dans l'école pour les soutenir. Toutefois, malgré l'intérêt que l'on peut avoir à documenter chacune de ces dimensions du climat scolaire interculturel, leur pertinence réelle réside dans les liens qu'elles peuvent avoir avec l'expérience scolaire des élèves et avec leur réussite éducative de manière plus spécifique.

\section{L’impact sur le climat interculturel sur la réussite éducative}

La contribution du climat scolaire général sur le rendement, l'engagement et la motivation des élèves est bien documentée (Crahay, 2012). Mais qu'en est-il de l'impact de chacune des dimensions du climat interculturel ? Les connaissances empiriques sur le sujet demeurent fragmentaires.

Concernant les liens entre le climat d'ouverture à la diversité dans l'école (dimension 1) et l'expérience scolaire des élèves, la recherche suggère que l'adoption de politiques dites d'éducation multiculturelle, interculturelle, antiraciste ou antidiscriminatoire dans les établissements scolaires est susceptible de jouer un rôle significatif, sinon direct, sur la réussite des élèves issus de l'immigration, du moins concernant leur intégration scolaire et leur sentiment d'appartenance à l'école (Christenson et Sheridan, 2001 ; Inglis, 2008 ; UNESCO, 2009). Selon certains auteurs, les élèves qui réussissent sont d'ailleurs les premiers à souligner qu'ils apprécient le fait que les politiques et les règles de leur école soient justes, équitables et qu'elles favorisent leur inclusion scolaire (Lafortune, 2012). Cependant, pour que de telles politiques d'ouverture puissent avoir un impact positif pour tous, il importe que l'école soit motivante et que les apprentissages véhiculés dans plusieurs cours et matières soient sensibles au vécu 
des élèves les plus vulnérables ou marginalisés (Mc Andrew et collab., 2015). La prise en compte de la diversité au sein de l'école ne doit donc pas se limiter à certains cours spécifiques, par exemple ceux d'éthique et de culture religieuse, ni favoriser une certaine élite, c'est-à-dire les élèves qui se conforment davantage aux demandes de l'école et qui réussissent le mieux, mais elle doit faire partie intégrante des valeurs et de la culture de l'ensemble du milieu.

De la même façon, la littérature scientifique nationale et internationale suggère que le degré d'ouverture de l'école aux langues et aux cultures des élèves (dimension 2) contribuerait significativement à l'amélioration de leur sentiment d'appartenance, de leur rapport à la scolarisation et de leur apprentissage de la langue du pays d'accueil. Pour les langues, les experts recommandent notamment aux écoles de favoriser l'émergence d'un " bilinguisme additif ", un principe selon lequel l'acquisition d'une langue seconde devient un outil de communication complémentaire qui s'ajoute au bagage de l'élève et qui n'implique pas le rejet de sa langue d'origine (Armand, Beck et Murphy, 2009). Ces constats induisent d'ailleurs que cette dimension du climat scolaire interculturel peut avoir un impact à plus long terme sur le cheminement éducatif des élèves (Christensen et Sheridan, 2001; Cummins, 2000 ; UNESCO, 2009), bien que la démonstration empirique de ces liens reste à faire.

Contrairement aux deux premières dimensions, les travaux concernant les attitudes du personnel à l'égard de la diversité (dimension 3) ou sur la qualité des relations interculturelles au sein de l'école (dimension 4) en lien avec la réussite éducative des élèves sont un peu plus nombreux. Des auteurs ont par exemple établi que lorsque les attitudes du personnel à l'égard de la diversité s'inscrivent dans une logique déficitaire qui met l'accent sur les difficultés des élèves et de leurs familles et qui est peu axée sur les facteurs systémiques qui relèvent de l'école, ces attitudes ont un effet néfaste évident sur le rendement des élèves (Agirdag, Van Avermaet et Van Houtte, 2013 ; Caprara, Barbaranelli, Steca et Malone 2006 ; Franson, 1999; García et Guerra, 2004 ; Muijs et Reynolds, 2003 ; Vollmer, 2000 ; Midgley, Feldlaufer et Eccles, 1989). De surcroît, certains auteurs soulignent que, bien que les acteurs scolaires œuvrant en contexte de diversité apprécient leur travail et se sentent généralement respectés, considérés et valorisés, ils rapportent néanmoins certains défis particuliers (Benoît, Rousseau, Ngirumpatse et Lacroix, 2008). La pression importante de certains parents sur l'école et sur leurs enfants pour que ces derniers aient d'excellentes notes et l'écart entre les représentations et valeurs du personnel scolaire et celles de certaines familles font partie des difficultés mentionnées. Par ailleurs, si les perceptions du personnel scolaire par rapport à la diversité sont susceptibles d'être influencées négativement par leurs inquiétudes concernant divers enjeux et débats sociaux, l'effet de leurs perceptions sur les pratiques effectives qu'ils utilisent auprès des élèves varie plutôt selon l'éthique professionnelle, la discipline enseignée ou l'importance accordée à la relation pédagogique et au bagage des élèves (Castro, 2010 ; Delruelle et Torfs, 2005 ; Hohl et Normand, 2000). On peut donc penser que le fait de permettre aux acteurs scolaires de mieux connaître les jeunes et leurs familles et de démystifier certains préjugés à leur égard est susceptible de les aider à se sentir plus efficaces, à développer des liens de qualité avec leurs élèves et, ainsi, à favoriser l'engagement et la réussite éducative de ces derniers (Archambault, Janosz, Dupéré, Brault et Mc Andrew, 2017 ; Izzo, Weissberg, Kasprow et Fendrich, 1999; Lefebvre et Legault, 2006 ; Suarez-Orozco, Pimentel et Martin, 2009).

Quant à la qualité des relations interculturelles au sein de l'école (dimension 4), les études nationales comme internationales insistent fortement sur l'importance de cette dimension sur la réussite éducative des élèves (Archambault, Janosz, Fallu et Pagani, 2009; Christenson, Reschly, Appleton, Berman, Spanjers et Varro, 2008; Suárez-Orozco et collab. 2009 ; Suárez-Orozco, Gaytán, Bang, Pakes, O’Connor et Rhodes, 2010). Au Québec, la recherche quantitative d'Archambault et collab. (2015) a confirmé l'influence positive du climat de sécurité et de justice, ainsi que la contribution négative des relations conflictuelles avec les enseignants sur l'engagement scolaire des élèves issus de l'immigration fréquentant une école de milieu défavorisé. L'auteure et ses collègues montrent également qu'au contraire, le fait de partager des relations chaleureuses avec un enseignant est associé positivement à l'engagement de ces élèves, particulièrement des garçons. De même, les données des études qualitatives qui abordent indirectement cet enjeu illustrent l'importance d'un climat de relations interculturelles positif pour les jeunes en situation de réussite scolaire, et particulièrement pour les élèves issus de groupes ethnoculturels reconnus par la recherche comme rencontrant davantage de difficultés (Bakhshaei, 2013; Mc Andrew et collab., 2004 ; Kanouté et Lafortune, 2011 ; Triki-Yamani et Mc Andrew, 2009 ; Vatz Laaroussi et collab., 2005). Enfin, d’autres travaux réalisés aux États-Unis vont dans le même sens et suggèrent que le 
développement de relations chaleureuses et positives avec un adulte au sein de l'école est fortement associé à l'engagement et à la réussite éducative des élèves issus de l'immigration, quelle que soit leur appartenance ethnoculturelle (Suarez-Orozco et collab., 2009).

Enfin, bien qu'il n'existe pas de lien univoque entre la performance scolaire et le niveau d'acculturation des élèves (Mc Mullen, 2009; Suárez-Orozco et Carhill, 2008), certains travaux suggèrent que le soutien de l'école à la construction identitaire (dimension 5) peut aussi contribuer à la réussite et plus largement à l'expérience socioscolaire positive des élèves. Au Québec, diverses études semblent montrer que la majorité des jeunes issus de l'immigration développent une identité positive basée sur un équilibre entre leurs cultures d'origine et d'accueil, et que ce profil identitaire serait généralement associé à la réussite scolaire (Bakhshaei, 2013; Harnois, 2010; Lafortune, 2006, 2012 ; Rahm, 2013 ; Triki-Yamani et Mc Andrew, 2009). Toutefois, ces mêmes études montrent que chez certains élèves, les conflits de valeurs non résolus ont un impact négatif sur leur rapport à l'école et leur persévérance scolaire, particulièrement chez les groupes appartenant à des communautés marginalisées dans la société. Les auteurs insistent donc sur l'importance pour les jeunes de se construire une identité harmonieuse favorisant leur réussite éducative et sur le rôle central que l'école peut jouer pour les soutenir dans ce processus. Le fait qu'il existe un écart entre les valeurs véhiculées par l'école et celles de certaines familles (par exemple, sur le rôle et le statut des hommes et des femmes ou la conception de la discipline et de l'autonomie des jeunes) ne devrait pas être posé comme un problème en soi. C'est plutôt l'interprétation, les attitudes et les messages qui sont envoyés par les acteurs scolaires par rapport à ces écarts qui peuvent favoriser ou nuire à la construction identitaire des élèves et, en retour, à leur réussite éducative. Ainsi, lorsque les conflits identitaires sont mal gérés par les jeunes ou mis en exergue dans l'école, à travers des commentaires ou des interventions qui incitent l'élève à choisir ou privilégier la culture du pays d'accueil au détriment des valeurs de sa culture d'origine ou de sa famille, ses besoins d'appartenance et d'identification demeurent bien souvent inassouvis. Au final, ce problème peut contribuer à augmenter l'aliénation, l'implication dans des réseaux d'amis qui présentent des comportements déviants ou, à l'extrême, favoriser la sympathie pour la radicalisation violente (Rousseau et collab., 2018 ; TardifGrenier, Archambault et Janosz, 2011). Cependant, ici encore, il y aurait lieu d'explorer cette dimension du climat scolaire interculturel et ses rapports avec la réussite éducative de manière beaucoup plus systématique, pour en favoriser une compréhension exhaustive et plus fine.

\section{Conclusion}

En somme, outre qu'elles sont en lien avec les trois sphères du climat identifiées par Moos (1979), les cinq dimensions du climat interculturel que nous proposons éclairent les différentes normes, valeurs et croyances qui caractérisent les écoles en contexte de diversité. La première dimension permet en effet d'appréhender le cadre scolaire dans une perspective systémique qui dépasse les marqueurs ethnoculturels, religieux et linguistiques pour s'intéresser à la façon dont la diversité, sous toutes ses formes, est prise en compte dans les encadrements de l'établissement, ainsi que dans les pratiques destinées aux élèves vulnérables. La seconde dimension, quant à elle, se centre davantage sur les pratiques d'enseignement-apprentissage préconisées en classe et à l'échelle de l'école, alors que les troisième et quatrième dimensions permettent de mieux comprendre le climat à partir de la prise en compte des relations interpersonnelles interculturelles. Finalement, la cinquième dimension proposée porte plus particulièrement sur l'expérience des élèves issus de la diversité en empruntant une perspective plus individuelle, qui renvoie à leur développement identitaire.

Les dimensions que nous proposons n'ont cependant pas le mérite de couvrir toutes les sphères du climat scolaire documentées dans les études antérieures. Par exemple, les climats de sécurité ou d'appartenance qui ont fait l'objet de bon nombre de travaux ne sont pas ici directement abordés. Ces dimensions sont plutôt traitées à travers les perceptions des élèves vis-à-vis de l'école et de la qualité des relations interculturelles. De surcroît, le climat de justice qui a également été proposé antérieurement comme une dimension importante du climat (Janosz et collab., 1998) est ici davantage traité de manière transversale, à travers le principe d'équité qui est central à toutes les dimensions.

Malgré ces limites, l'examen systémique des cinq dimensions que nous proposons met l'accent sur la responsabilité que doit avoir l'école pour favoriser l'équité et l'inclusion scolaire de tous ses élèves (Inglis, 2008; Ministère de 
l'Éducation du Québec [MEQ], 1998). Un portrait plus exhaustif des dimensions du climat scolaire interculturel et de leurs manifestations dans différents milieux s'impose donc, tout comme une meilleure compréhension du rôle de ces dimensions sur la réussite éducative des élèves de toutes origines. II s'agit en effet d'étapes incontournables, tant pour la mise en œuvre d'encadrements, de politiques et de programmes équitables et inclusifs que pour l'actualisation de la pratique des acteurs scolaires qui travaillent auprès d'élèves issus de la diversité. Dans une perspective de justice sociale, cette prise en compte ne peut que soutenir l'adoption de pratiques plus inclusives, valorisant la diversité sous toutes ses formes et tenant compte des besoins de tous les élèves, favorisant ainsi davantage l'égalité des chances pour tous de réussir.

\section{Références bibliographiques}

Abou, S. (1986). L'identité culturelle. Relations interethniques et problèmes d'acculturation. Paris: Anthropos.

Agirdag, O., Van Avermaet, P. et Van Houtte, M. (2013). School segregation and math achievement: A mixedmethod study on the role of self-fulfilling prophecies. Teachers College Record, 115(3), 1-50.

Alspaugh, J.W. (1998). Achievement loss associated with the transition to middle school and high school. The Journal of Educational Research, 92(1), 20-25.

Amiraux, V., (2018). Comment parler du pluralisme religieux ? Dans D. Heimpel, S. Taher (dir.), Les défis du pluralisme : regards croisés Europe-Amérique du Nord (p. 125-135). Montréal : Presses de l'Université de Montréal.

Archambault, I., Janosz, M., Fallu, J. S. et Pagani, L. S. (2009). Student engagement and its relationship with early high school dropout. Journal of Adolescence, 32(3), 651-670.

Archambault, I., Janosz, M., Dupéré, V., Brault, M-C. et Mc Andrew, M. (2017). Individual, social, and family factors associated with high school dropout among low-SES youth: Differential effects as a function of immigrant status. British Journal of Educational Psychology, 87(3), 456-477

Archambault, I., Tardif-Grenier, K., Dupéré, V., Janosz, M., Mc Andrew, M., Pagani, L., Parent, et collab. (2015). Étude comparative de l'engagement scolaire des élèves de milieux défavorisés issus ou non de l'immigration : contributions de l'environnement scolaire et des pratiques enseignantes. Rapport soumis au Fonds de recherche Société et culture, septembre 2015.

Armand, F. (2013). Accompagner les milieux scolaires : les enjeux de la prise en compte de la diversité linguistique en contexte montréalais francophone. Dans M. Mc Andrew, M. Potvin et C. Borri-Anadon (dir.), Le développement d'institutions inclusives en contexte de diversité. Recherche, formation, partenariat (p. 137152). Montréal : Presses de l'Université du Québec.

Armand, F. et Maraillet, É. (2013). Éducation interculturelle et diversité linguistique. Montréal : Université de Montréal, Elodil (Éveil au Langage et Ouverture à la Diversité Linguistique). http://www.elodil.umontreal.ca/guides/education-interculturelle-et-diversite-linguistique/

Armand, F., Hardy, M.H. et Lemay, B. (2014). Le soutien en langue maternelle : une approche novatrice pour les élèves allophones en difficulté ! Québec français, 172, 23-24. https://id.erudit.org/iderudit/72005ac

Armand, F., Beck, I. A. et Murphy, T. (2009). Réussir l'intégration des élèves allophones immigrants nouvellement arrivés. Vie Pédagogique, 152, 106-115. http://collections.banq.qc.ca/ark:/52327/bs1963884

Audet, G., Mc Andrew, M. et Lamothe-Lachaine, A. (soumis). Relations école-famille immigrante-communauté : pratiques, projets et pistes d'action pour le milieu scolaire.

Bakhshaei, M. (2013). L'expérience socioscolaire d'élèves montréalais originaires de l'Asie du Sud : dynamiques familiales, communautaires et systémiques. (Thèse de doctorat, Université de Montréal, Montréal). https://papyrus.bib.umontreal.ca/xmlui/handle/1866/10117

Benoit, M., Rousseau, C., Ngirumpatse, P. et Lacroix, L. (2008). Relations parents immigrants-écoles dans l'espace montréalais : au-delà des tensions, la rencontre des rêves. Revue des sciences de l'éducation, 34(2), 313-332. 
Bingham, G. E. et Okagaki, L. (2012). Ethnicity and student engagement. Dans S. L. Christenson, A. L. Reschly et C. Wylie (dir.), Handbook of research on student engagement (p. 65-95). New York : Springer.

Borri-Anadon, C. (2014). Pratiques évaluatives des orthophonistes à l'égard des élèves issus de minorités culturelles : une recherche interprétative critique (Thèse de doctorat, Université du Québec à Montréal, Montréal). https://archipel.uqam.ca/6351/

Borri-Anadon, C. et Hirsch, S. (2018). Des clés pour mieux comprendre la diversité ethnoculturelle, religieuse et linguistique en milieu scolaire: fiches régionales. Trois-Rivières : Université du Québec à Trois-Rivières, en collaboration avec la Direction des services d'accueil et d'éducation interculturelle du Ministère de l'éducation et de l'enseignement supérieur. http://ofde.ca/wp-content/uploads/2017/10/MauricieVF.pdf

Bradshaw, C. P., Koth, C. W., Thornton, L. A. et Leaf, P. J. (2009). Altering school climate through school-wide Positive Behavioral Interventions and Supports: Findings from a group-randomized effectiveness trial. Prevention Science, 10(2), 100-115.

Brault, M. C., Janosz, M. et Archambault, I. (2014). Effects of school composition and school climate on teacher expectations of students: A multilevel analysis. Teaching and Teacher Education, 44, 148-159.

Camilleri, C. (1990). Identité et gestion de la disparité culturelle : essai d’une typologie. Dans C. Camilleri, J., Kastersztein, E.M., Lipiansky, H., Malewska-Peyre, I., Taboada-Leonetti et A. Vasquez-Bronfman (dir.), Stratégies identitaires (p. 85-110). Paris : Presses Universitaires de France.

Caprara, G. V., Barbaranelli, C., Steca, P. et Malone, P. S. (2006). Teachers' self-efficacy beliefs as determinants of job satisfaction and students' academic achievement: A study at the school level. Journal of School Psychology, 44(6), 473-490.

Castro, A.J. (2010). Themes in the research on preservice teachers' views of cultural diversity: Implications for researching millennial preservice teachers. Educational Researcher, 39(3), 198-210.

Chiu, M. M., Pong, S. L., Mori, I. et Chow, B. W. (2012). Immigrant students' emotional and cognitive engagement at school: A multilevel analysis of students in 41 countries. Journal of Youth and Adolescence, 41(11), 14091425

Christenson, S.L. et Sheridan, S.M. (2001). School and families: Creating essential connections for learning. New York : Guilford Press.

Christenson, S.L., Reschly, A.L., Appleton, J.J., Berman, S., Spanjers, D. et Varro, P. (2008). Best practices in fostering student engagement. Dans A. Thomas et J. Grimes (dir.), Best Practices in School Psychology, $\left(5^{\mathrm{e}}\right.$ éd, p. 10991120). Washington, DC : National Association of School Psychologists.

Cohen, J., McCabe, L., Michelli, N. M. et Pickeral, T. (2009). School climate: Research, policy, practice, and teacher education. Teachers College Record, 111(1), 180-213.

Comité consultatif sur l'intégration et l'accommodement raisonnable en milieu scolaire (CCIARMS). (2007). Une école québécoise inclusive : dialogue, valeurs et repères communs. Québec : Ministère de l’Éducation, du Loisir et du Sport.

http://www.education.gouv.qc.ca/fileadmin/site_web/documents/dpse/formation_jeunes/RapportAccRais onnable.pdf

Conseil supérieur de l'éducation. (2017). Pour une école riche de tous ses élèves. S'adapter à la diversité des élèves, de la maternelle à la $5^{e}$ année du secondaire. Québec : Gouvernement du Québec. http:/cse.gouv.qc.ca/fichiers/documents/publications/Avis/50-0500.pdf

Crahay, M. (2012). L'école peut-elle être juste et efficace? De l'égalité des chances à l'égalité des acquis. Liège : De Boeck.

Cummins, J. (2000). Language, power and pedagogy: Bilingual children in the crossfire. Clevedon, Royaue-Uni : Multilingual Matters. 
D’Amato, J. (1993). Resistance and compliance in minority classrooms. Dans E. Jacob et C. Jordan (dir.), Minority education: Anthropological perspectives (p. 181-207). Norwood, NJ: Ablex.

De Koninck, Z. et Armand, F. (2012). Portrait des services d'accueil et d'intégration scolaire des élèves issus de l'immigration. Québec : Ministère de l’Éducation, du Loisir et du Sport. https://www.ceetum.umontreal.ca/fileadmin/documents/publications/2012/de-koninck-armand2012.pdfM

Dee, T. S. (2004). Teachers, race, and student achievement in a randomized experiment. The Review of Economics and Statistics, 86(1), 195-210.

Delruelle, E. et Torfs, R. (2005). Commission du dialogue interculturel (Rapport final). Bruxelles : Centre pour l'égalité des chances et la lutte contre le racisme.

https://www.unia.be/files/Documenten/Publicaties_docs/Rapport_final_Commission_du_dialogue_intercul turel.pdf

Ewing, A. R. et Taylor, A. R. (2009). The role of child gender and ethnicity in teacher-child relationship quality and children's behavioral adjustment in preschool. Early Childhood Research Quarterly, 24(1), 92-105.

Faircloth, B. S. et Hamm, J. V. (2005). Sense of belonging among high school students representing four ethnic groups. Journal of Youth and Adolescence, 34(4), 293-309.

Franson, C. (1999). Mainstreaming learners of English as an additional second language: The class teachers perspective. Language, Culture and Curriculum, 12(1), 59-71.

Fuligni, A. J. (1997). The academic achievement of adolescents from immigrant families: The role of family background, attitudes, and behavior. Child Development, 68(2), 351-363.

García, S. B. et Guerra, P. L. (2004). Deconstructing deficit thinking: Working with educators to create more equitable learning environments. Education and Urban Society, 36(2), 150-168.

Garcia-Reid, P. (2007). Examining social capital as a mechanism for improving school engagement among low income Hispanic girls. Youth and Society, 39(2), 164-181.

Garcia-Reid, P., Reid, R. J. et Peterson, N. A. (2005). School engagement among Latino youth in an urban middle school context: Valuing the role of social support. Education and Urban Society, 37(3), 257-275.

Gélinas, K., Hirsch, S., et Borri-Anadon, C. (soumis). Les fiches régionales sur la diversité ethnoculturelle, religieuse et linguistique au Québec : un outil fécond pour l'enseignement de l'univers social.

Glock, S., Kneer, J. et Kovacs, C. (2013). Preservice teachers' implicit attitudes toward students with and without immigration background: A pilot study. Studies in Educational Evaluation, 39(4), 204-210.

Harnois L. (2010). Le partenariat école-famille-communauté : points de vue de parents réfugiés ou immigrants de Joliette, Lanaudière. Québec : Ministère de l’Éducation, du Loisir et du Sport.

Heath, S. B. (1983). Ways with words: Language, life, and work in communities and classrooms. New York: Cambridge University Press.

Hirsch, S. et Mc Andrew, M. (2013). Le traitement du judaïsme dans les manuels scolaires d'éthique et culture religieuse au Québec contribue-t-il à un meilleur vivre ensemble ? McGill Journal of Education / Revue des sciences de l'éducation de McGill, 48(1), 99-114.

Hohl, J. et Normand, M. (1996). Construction et stratégies identitaires des enfants et des adolescents en contexte migratoire : le rôle des intervenants scolaires. Revue Française de Pédagogie, 117, 39-52.

Hohl, J. et Normand, M. (2000). Enseigner en milieu pluriethnique dans une société divisée. Dans M. Mc Andrew et F. Gagnon (dir.), Relations ethniques et éducation dans les sociétés divisées, Québec, Irlande du Nord, Catalogne et Belgique, (p. 169-181). Paris/Montréal : L'Harmattan.

Inglis, C. (2008). Planning for cultural diversity. Paris : UNESCO. 
Izzo, C. V., Weissberg, R. P., Kasprow, W. J. et Fendrich, M. (1999). A longitudinal assessment of teacher perceptions of parent involvement in children's education and school performance. American Journal of Community Psychology, 27(6), 817-839.

Janosz, M., Georges, P. et Parent, S. (1998). L'environnement éducatif à l'école secondaire : un modèle théorique pour guider l'évaluation du milieu. Revue Canadienne de Psycho-Éducation, 27(2), 285-306.

Jia, Y., Way, N., Ling, G., Yoshikawa, H., Chen, X., Hughes, D., Ke, X. et Lu, Z. (2009). The influence of student perceptions of school climate on socioemotional and academic adjustment: A comparison of Chinese and American adolescents. Child Development, 80(5), 1514-1530.

Kanouté, F. et Lafortune, G. (2011). La réussite scolaire des élèves d’origine immigrée : réflexions sur quelques enjeux à Montréal. Éducation et Francophonie, 39(1), 80-92.

Konishi, C., Miyazaki, Y., Hymel, S. et Waterhouse, T. (2017). Investigating associations between school climate and bullying in secondary schools: Multilevel contextual effects modeling. School Psychology International, 38(3), 240-263.

Kuperminc, G. P., Leadbeater, B. J., Emmons, C. et Blatt, S. J. (1997). Perceived school climate and difficulties in the social adjustment of middle school students. Applied Developmental Science, 1(2), 76-88.

Lafortune, G. (2006). Vécu scolaire et stratégies identitaires d'adolescents montréalais d'origine haïtienne de première et de deuxième générations (Mémoire de maîtrise, Université de Montréal, Montréal). https://papyrus.bib.umontreal.ca/xmlui/bitstream/handle/1866/17682/Lafortune_Gina_2006_memoire.pdf

Lafortune, G. (2012). Rapport à l'école et aux savoirs scolaires de jeunes d'origine haïtienne en contexte scolaire défavorisé à Montréal (Thèse de doctorat, Université de Montréal, Montréal). https://papyrus.bib.umontreal.ca/xmlui/bitstream/handle/1866/8479/Lafortune_Gina_2012_these.pdf

LaFromboise, T., Coleman, H. L., et Gerton, J. (1993). Psychological impact of biculturalism: Evidence and theory. Psychological Bulletin, 114(3), 395-412.

Lamanque-Bélanger, C. (2018). La relation entre les interactions positives avec l'enseignant et les pairs sur l'engagement affectif des élèves du primaire immigrants et non-immigrants en milieux défavorisés (Mémoire de maîtrise, Université de Montréal, Montréal). https://papyrus.bib.umontreal.ca/xmlui/handle/1866/21303

Larochelle-Audet J., Magnan M. O., Potvin M. et Doré E. (dir.). (2018). Les compétences des directions en matière d'équité et de diversité : pistes pour les cadres de référence et la formation. Montréal : Université du Québec à Montréal (UQAM), Observatoire sur la formation à la diversité et l'équité (OFDE).

La Salle, T. P. (2018). International perspectives of school climate. School Psychology International, 39(6), 559-567.

La Salle, T. P., George, H. P., McCoach, B., Polk, T. et Evanovich, L. L. (2018). An Examination of school climate, victimization, and mental health problems among middle school students self-identifying with emotional and behavioral disorders. Behavioral Disorders, 43(3), 383-392.

La Salle, T. P., Meyers, J., Varjas, K. et Roach, A. (2015). A cultural-ecological model of school climate. International Journal of School and Educational Psychology, 3(3), 157-166.

Leadbeater, B. J., Sukhawathanakul, P., Thompson, K. et Holfeld, B. (2015). Parent, child, and teacher reports of school climate as predictors of peer victimization, internalizing and externalizing in elementary school. School Mental Health, 7(4), 261-272.

Lefebvre, M-L., Legault, F. (2006). Connaissance et assignation de stéréotypes de genre dans une population scolaire de pré-adolescents et pré-adolescentes de divers groupes ethnolinguistiques montréalais, La revue internationale de l'éducation familiale, 19(1), 77-106.

Livingstone, A. M., Celemencki, J. et Calixte, M. (2014). Youth participatory action research and school improvement. The missing voices of Black youth in Montreal. Canadian Journal of Education / Revue canadienne de l'éducation, 37(1), 283-307. 
Loukas, A., et Robinson, S. (2004). Examining the moderating role of perceived school climate in early adolescent adjustment. Journal of Research on Adolescence, 14(2), 209-233.

Lucas, S.R. (2001). Effectively maintained inequality: Education transitions, track mobility and social background effects. American Journal of Sociology, 106(6), 1642-1690.

Marks, A. K., Patton, F. et Coll, C. G. (2011). Being bicultural: A mixed-methods study of adolescents' implicitly and explicitly measured multiethnic identities. Developmental Psychology, 47(1), 270-288.

Mc Andrew, M. (2001). Immigration et diversité à l'école. Le débat québécois dans une perspective comparative. Montréal : Presses de l’Université de Montréal.

Mc Andrew, M. et Audet, G. (sous presse). L'immigration et la diversité ethnoculturelle dans les écoles québécoises : les grands encadrements, les programmes et les débats. Dans F. Lorcerie, A. Manço, M. Potvin et M. Sanchez-Mazas (dir.), Éducation et diversité. Les fondamentaux de l'action. Rennes : Presses universitaires de Rennes.

Mc Andrew, M., Balde, A., Bakhshaei, M., Tardif-Grenier, K., Audet, G., Armand, F., ... Rousseau, C. (2015). La réussite éducative des élèves issus de l'immigration. Dix ans de recherche et d'intervention au Québec. Montréal : Presses de l'Université de Montréal.

Mc Andrew, M., Éphraïm, S., Lemire, F. et Swift, M. (2004). La réussite scolaire des jeunes Noirs anglophones dans les écoles de langue française à Montréal : un bilan. Rapport de recherche soumis au ministère du Patrimoine canadien, Montréal.

McMullen, K. (2009). Self-Reported Delinquency of Immigrant Youth, Toronto 2006. Ottawa : Ministère de l'industrie.

McNeely, C. A., Nonnemaker, J. M. et Blum, R. W. (2002). Promoting school connectedness: Evidence from the national longitudinal study of adolescent health. Journal of School Health, (72)4, 138-146.

Midgley, C., Feldlaufer, H. et Eccles, J. S. (1989). Student/teacher relations and attitudes toward mathematics before and after the transition to junior high school. Child Development, 60(4), 981-992.

Milot, M. (2014). École et religions au Québec : principes en débat et pratiques en questionnement. Histoire, Monde et Cultures Religieuses, 32, 55-66.

Ministère de l'Éducation du Québec. (1998). Une école d'avenir : la politique d'intégration scolaire et d'éducation interculturelle. Québec : Gouvernement du Québec.

Miranda, A. O., Estrada, D. et Firpo-Jimenez, M. (2000). Differences in family cohesion, adaptability, and environment among Latino families in dissimilar stages of acculturation. The Family Journal: Counseling and Therapy for Couples and Families, 8(4), 341-350.

Moos, R. H. (1979). Evaluating educational environments: Procedures, measures, findings, and policy implications. San Francisco : Jossey-Bass.

Morrissette, J. et Demazière, D. (2018). Dualité des processus de socialisation professionnelle des enseignants formés hors Québec : entre imposition et appropriation, Alterstice, 8(1), 95-106.

Morrissette, J., Diédhiou, S. B. M. et Charara, Y. (2014). Un portrait de la recherche sur l'intégration socioprofessionnelle des enseignants formés à l'étranger. Rapport de recherche déposé au Centre d'intervention pédagogique en contexte de diversité de la Commission scolaire Marguerite-Bourgeoys, Montréal.

Muijs, D. et Reynolds, D. (2003). Student background and teacher effects on achievement and attainment in mathematics: A longitudinal study. Educational Research and Evaluation, 9(3), 289-314.

Murphy, T. (2014). Représentations d'enseignants quant à l'intégration linguistique, socioscolaire, scolaire et sociale d'élèves allophones immigrants dans trois écoles secondaires montréalaises (Mémoire de maîtrise, Université de Montréal, Montréal). https://papyrus.bib.umontreal.ca/xmlui/handle/1866/11886 
Niyubahwe, A., Mukamurera, J. et Jutras, F. (2013). Professional integration of immigrant teachers in the school system: A literature review. Revue des sciences de l'éducation de McGill, 48(2), 279-296.

Oueslati, B., Mc Andrew, M. et Helly, D. (2011). Islam and Muslim cultures in Quebec French-language textbooks over three periods: 1980s, 1990s, and the present day. Journal of Educational Media, Memory, and Society, $3(1), 5-24$.

Parris, L., Rocha Neves, J. et La Salle T. (2018). School climate perceptions of ethnically diverse students: Does school diversity matter? School Psychology International, 39(6), 625-645.

Piet, G. et Grandjean, G. (dir.), (2012). L'école du vivre-ensemble. Paris : Armand Colin.

Potvin, M., Larochelle-Audet, J., Campbell, M.-È., Kingué-Élonguélé, G. et Chastenay, M.-H. (2015). Revue de littérature sur les compétences en matière de diversité ethnoculturelle, religieuse et linguistique dans la formation du personnel scolaire, selon différents courants théoriques. Montréal, Canada : Observatoire sur la formation à la diversité et l'équité (OFDE). Récupéré de : http://www.ceetum.umontreal.ca/documents/publications/2015/revue-competences-diversite.pdf

Rahm, J. (2013). After school and community programs' role in supporting immigrant youth: Stories of opportunities for youths' identity work and learning for life. Dans N. Trépanier (dir.), Plaidoyer pour une école communautaire/Making the case for community schools (p.87-106). Montréal : Éditions Nouvelles.

Roscigno, V. J. et Ainsworth-Darnell, J. W. (1999). Race, cultural capital, and educational resources: Persistent inequalities and achievement returns. Sociology of Education, 72(3), 158-178.

Rousseau, C., Hassan, G., Rousseau-Rizzi, A., Michalon-Brodeur, V., Oulhote, Y., Mekki-Berrada, A. et El-Hage, H. (2018). Adversité psychosociale, détresse psychologique et sympathie pour la radicalisation violente chez les collégiens du Québec. Cahiers de la Sécurité et de la Justice, 43, 158-166.

Ryan, A. M. (2000). Peer groups as a context for the socialization of adolescents' motivation, engagement and achievement in school. Educational Psychologist, 35(2), 101-111.

Sanders, S. M., Durbin, J. M., Anderson, B. G., Fogarty, L. M., Giraldo-Garcia, R. J. et Voight, A. (2018). Does a rising school climate lift all boats? Differential associations of perceived climate and achievement for students with disabilities and limited English proficiency. School Psychology International, 39(6), 646-662.

Sharkey, J. et Layzer, C. (2000). Whose definition of success? Identifying factors that affect English language learners' access to academic success and resources. TESOL Quarterly, 34(2), 252-368.

Smokowski, P. R., Rose, R. et Bacallao, M. L. (2008). Acculturation and latino family processes: How cultural involvement, biculturalism, and acculturation gaps influence family dynamics. Family Relations, 57, $295-308$.

Suárez-Orozco, C. et Carhill, A. (2008). Afterword: New directions in research with immigrant families and their children. New Directions for Child and Adolescent Development, 121, 87-104.

Suárez-Orozco, C., Gaytán, F. X., Bang, H. J., Pakes, J., O’Connor, E. et Rhodes, J. (2010). Academic trajectories of newcomer immigrant youth. Developmental Psychology, 46(3), 602-618

Suárez-Orozco, C., Pimentel, A. et Martin, M. (2009). The significance of relationships: Academic engagement and achievement among newcomer immigrant youth. Teachers College Record, 111(3), 712-749.

Suárez-Orozco, C., Rhodes, J. et Milburn, M. (2009). Unraveling the immigrant paradox. Academic engagement and disengagement among recently arrived immigrant youth. Youth and Society, 41(2), 151-185.

Tardif-Grenier, K., Archambault I. et Janosz, M. (2011). Les pratiques parentales, le désengagement scolaire des amis et le rendement scolaire chez les élèves du secondaire nés à Haïti et fréquentant une école en milieu défavorisé. Revue de Psychoéducation, 40(2), 261-282.

Tardif-Grenier, K., Archambault, I., Lafantaisie, V. et Magnan, M. O. (2018). La collaboration école-famille immigrante : représentations d'administrateurs en milieu scolaire primaire socioéconomiquement défavorisé. Revue Canadienne de l'Éducation, 41(4), 954-990. 
Thamin, N. (2011). Constructions identitaires d'acteurs sociaux plurilingues en contexte migratoire français : quelques éléments de réflexion pour une reconnaissance de la pluralité linguistique et culturelle. Cahiers de I'ILOB (Individual Plurilingualism and Multilingual Communities in a Context of Official Bilingualism), 2(1).

Timotijevic, L. et Breakwell, G. M. (2000). Migration and threat to identity. Journal of Community \& Applied Social Psychology, 10(5), 355-372.

Triki-Yamani, A. et Mc Andrew, M. (2009). Perceptions du traitement de l'islam, du monde musulman et des minorités musulmanes par de jeunes musulmans(es) du cégep au Québec. Diversité urbaine, 9(1), 73-94.

UNESCO. (2009). Principes directeurs pour l'inclusion dans l'éducation. Paris : UNESCO.

Vaquera, E. (2009). Friendship, educational engagement, and school belonging: Comparing Hispanic and White adolescents. Hispanic Journal of Behavioral Sciences, 31(4), 492-514.

Vatz Laaroussi, M., Rachédi, L., Kanouté, F. et Duchesne, K. (2005). Favoriser les collaborations famille immigrantes/école en soutien à la réussite scolaire. Guide d'accompagnement. Sherbrooke : Université de Sherbrooke.

Vedder, P., Boekaerts, M. et Seegers, G. (2005). Perceived social support and well being in school; The role of students' ethnicity. Journal of Youth and Adolescence, 34(3), 269-278.

Voight, A., Hanson, T., O'Malley, M. et Adekanye, L. (2015). The racial school climate gap: Within-school disparities in students' experiences of safety, support and connectedness. American Journal of Community Psychology, 56(3-4), 252-267.

Vollmer, G. (2000). Praise and stigma: Teachers' constructions of the typical ESL students. Journal of Intercultural Studies, 21(1), 53-66.

Wang, C., Boyanton, D., Ross, A. M., Liu, J. L., Sullivan, K. et Do, K. A. (2018). School climate, victimization, and mental health outcomes among elementary school students in China. School Psychology International, 39(6), 587-605.

Watkins, N. D. et Aber, M. S. (2009). Exploring the relationships among race, class, gender, and middle school students' perceptions of school racial climate. Equity and Excellence in Education, 42(4), 395-411.

Way, N., Becker, B. E. et Greene, M. L. (2006). Friendships among Black, Latino, and Asian American adolescents in an urban context. Dans L. Balter et C. S. Tamis-LeMonda (dir.), Child psychology: A handbook of contemporary issues (p. 415-443). New York : Psychology Press.

White, N., La Salle, T., Ashby, J. S. et Meyers, J. (2014). A brief measure of adolescent perceptions of school climate. School Psychology Quarterly, 29(3), 349-359. https://psycnet.apa.org/doi/10.1037/spq0000075 